Enferm Bras 2021;20(1);94-108

doi: 10.33233/eb.v20i1.4438

\title{
REVISÃo
}

\section{Complicações associadas a COVID-19 e as principais necessidades humanas básicas afetadas}

Josiele de Lima Neves, M.Sc. ${ }^{*}$ Eda Schwartz, D.Sc. ${ }^{* *}$, Lílian Moura de Lima Spagnolo, D.Sc. ${ }^{\star \star \star}$, Julia Peres Ávila ${ }^{\star \star \star}$, Naiane Pereira de Oliveira ${ }^{\star \star \star \star *}$, Fernanda Lise, D.Sc. ${ }^{* \star \star \star *}$

*Enfermeira, Doutoranda do Programa de Pós-Graduação em Enfermagem da UFPel, Pelotas/RS, Bolsista CAPES, "*Enfermeira, Docente da Faculdade de Enfermagem (FEn) e do Programa de Pós-Graduação da Faculdade de Enfermagem e do Programa de Mestrado Profissional em Saúde da Família da UFPel, Pelotas/RS, ${ }^{* * *}$ Enfermeira, Professora da Faculdade de Enfermagem e do Programa de Pós-Graduação da Faculdade de Enfermagem da UFPel, Pelotas/RS, ${ }^{* * * *}$ Graduanda em enfermagem pela UFPel, Pelotas/RS, ${ }^{\star * \star * \star}$ Graduanda em enfermagem pela UFPEL, Pelotas/RS, ${ }^{\star * \star \star * \star}$ Enfermeira, Membro do comitê de práticas com famílias da International Family Nursing Association (IFNA) e do Núcleo de Condições Crônicas e suas Interfaces (NUCCRIN) da Faculdade de Enfermagem da UFPel

Recebido em 4 de novembro de 2020; aceito em 5 de janeiro de 2020.

Correspondência: Josiele de Lima Neves, Rua Maestro Medanha, 550/403, BI 6, 96020-720 Pelotas RS

Josiele de Lima Neves: josiele_neves@hotmail.com

Eda Schwartz: edaschwa@gmail.com

Lílian Moura de Lima Spagnolo: lima.lilian@gmail.com

Julia Peres Ávila: juu.peres11@gmail.com

Naiane Pereira de Oliveira: nah3m@hotmail.com

Fernanda Lise: fernandalise@gmail.com 


\section{Resumo}

Introdução: Os pacientes infectados pela COVID-19 possuem mecanismos de defesa do sistema imunológico ainda duvidoso e sem tratamento médico definitivo, portanto, é prudente que os profissionais da área da saúde estejam atentos a minuciosos sinais de piora em toda sintomatologia. Objetivo: Objetivouse conhecer as complicações associadas ao novo coronavírus e, por meio delas, elencar as principais necessidades humanas básicas segundo a Teoria das Necessidades Humanas Básicas. Métodos: Revisão integrativa da literatura através da Biblioteca Virtual Scientific Electronic Library Online (Scielo) Sistema Latino-Americano e do Caribe de Informação em Ciências da Saúde (Lilacs); National Library of Medicine (Pubmed), e na Cochrane. Foram utilizados os descritores 'Respiratory Distress Syndrome', 'Nursing' e 'COVID-19', com o operador boleano 'AND', sendo os mesmos previamente consultados nos dicionários Medical (MeSH) e Descritores em Ciências da Saúde (DeCS). Resultados: Fizeram parte do estudo oito publicações que trouxeram como complicações do novo coronavírus as infecções respiratórias, complicações cardiovasculares e relatos de acompanhamentos de pacientes sintomáticos, através das quais foi possível identificar as necessidades humanas básicas psicobiológicas, e psicossociais afetadas. Conclusão: Ferramentas como a Teoria das Necessidades Humanas Básicas podem contribuir para a viabilização de um cuidado efetivo ao paciente, o que possibilita uma assistência integral.

Palavras-chave: Enfermagem; cuidados críticos; tomada de decisão; síndrome do desconforto respiratório do adulto.

\section{Abstract}

Complications associated with COVID-19 and the main affected basic human needs

Introduction: Patients infected with COVID-19 have immune defense mechanisms that are still in doubt and without definitive medical treatment, so it is prudent for health professionals to be attentive to detailed signs of worsening in all symptoms. Objective: The objective was to know the complications associated with the new coronavirus and, through them, list the main basic human needs according to the Theory of Basic Human Needs. Methods: Integrative literature review through the Virtual Library Scientific Electronic 
Library Online (Scielo) Latin American and Caribbean Health Sciences Information System (Lilacs); National Library of Medicine (Pubmed), and Cochrane. The descriptors 'Respiratory Distress Syndrome', 'Nursing' and 'COVID-19' were used, with the Boolean operator 'AND', which were previously consulted in the Medical (MeSH) and Health Science Descriptors (DeCS) dictionaries. Results: Eight publications were part of the study, which brought respiratory infections, cardiovascular complications and follow-up reports of symptomatic patients as complications of the new coronavirus, through which it was possible to identify the basic human psychobiological and psychosocial needs affected. Conclusion: Tools such as the Theory of Basic Human Needs can contribute to enabling effective patient care, which enables comprehensive care.

Keywords: Nursing; critical care; decision making; respiratory distress syndrome; adult.

\section{Resumen}

Complicaciones asociadas con COVID-19 y las principales necesidades humanas básicas afectadas

Introducción: Los pacientes infectados por COVID-19 tienen mecanismos de defensa inmunológica aún en duda y sin tratamiento médico definitivo, por lo que es prudente que los profesionales de la salud estén atentos a los signos detallados de agravamiento de todos los síntomas. Objetivo: El objetivo era conocer las complicaciones asociadas al nuevo coronavirus y, a través de ellas, enumerar las principales necesidades humanas básicas según la Teoría de las Necesidades Humanas Básicas. Métodos: Revisión integradora de la literatura a través de la Biblioteca Virtual Biblioteca Científica Electrónica en Línea (Scielo) Sistema de Información en Ciencias de la Salud de América Latina y el Caribe (Lilacs); Biblioteca Nacional de Medicina (Pubmed) y Cochrane. Se utilizaron los descriptores "Respiratory Distress Syndrome", "Nursing" y "COVID-19", con el operador booleano "AND", que fueron consultados previamente en los diccionarios Medical (MeSH) y Health Science Descriptors (DeCS). Resultados: Ocho publicaciones formaron parte del estudio, que trajeron infecciones respiratorias, complicaciones cardiovasculares e informes de seguimiento de pacientes sintomáticos como complicaciones del nuevo coronavirus, a través de 
los cuales fue posible identificar las necesidades psicobiológicas y psicosociales humanas básicas afectadas. Conclusión: Herramientas como la Teoría de las Necesidades Humanas Básicas pueden contribuir a posibilitar una atención eficaz al paciente, que posibilita una atención integral.

Palabras-clave: Enfermería; cuidados críticos; toma de decisiones; síndrome de dificultad respiratoria del adulto.

\section{Introdução}

Os pacientes infectados pela COVID-19 possuem mecanismos de defesa do sistema imunológico ainda duvidoso e sem tratamento médico definitivo, portanto, é prudente que os profissionais da área da saúde estejam atentos a minuciosos sinais de piora em toda sintomatologia [1]. O comprometimento respiratório é uma das complicações mais evidentes, que pode evoluir rapidamente para a Síndrome Respiratória Aguda Grave (SARS-CoV), evidenciada como uma condição grave de comprometimento pulmonar, associada a Lesão Pulmonar Aguda (LPA), que se caracteriza pela complacência pulmonar reduzida, hipoxemia, devido às alterações de ventilação/perfusão do parênquima pulmonar, o que causa hipoxemia grave e infiltrações pulmonares bilaterais, acusados em radiografias [2,3].

Considerando a disseminação acentuada da doença é prudente identificar as principais complicações da COVID-19 para auxiliar os profissionais na identificação e manejo adequado em cada caso, proporcionando uma melhor resposta ao combate da pandemia [4]. Neste contexto, a atuação do enfermeiro pode contribuir para compreender as necessidades do indivíduo e permear estratégias para sistematizar o cuidado.

A Sistematização da Assistência de Enfermagem (SAE) é a organização do trabalho da enfermagem, com determinação de uma metodologia ou Teoria de Enfermagem e instrumentos para a implementação do Processo de Enfermagem (PE), dividido em cinco etapas: Histórico, Diagnóstico, Planejamento, Implementação e Avaliação de Enfermagem [5]. O PE é realizado através de ações sistematizadas e interrelacionadas, com a intenção de prestar 
assistência ao ser humano, responsáveis por um processo de raciocínio e julgamento clínico capaz de orientar as ações da Enfermagem [6,7].

Uma das teorias comumente utilizadas para o emprego da SAE e que orienta as etapas do Processo de Enfermagem em instituições brasileiras é a Teoria das Necessidades Humanas Básicas (NHB), criada e fundamentada por Wanda Horta [8]. Horta classificou as NHB pelos níveis de vida psíquica de João Mohana, os quais são divididos em psicobiológico, psicossocial e psicoespiritual, os quais possuem componentes inconscientes e que tendem à ser espontâneo ou de necessidades fundamentais da natureza humana [7]. Contudo, este estudo objetivou conhecer as complicações associadas a COVID-19 e, por meio delas, elencar as necessidades humanas básicas afetadas segundo a Teoria das Necessidades Humanas Básicas.

\section{Métodos}

Recorreu-se a revisão integrativa da literatura, que possibilita a síntese e análise do conhecimento científico já produzido sobre o tema investigado [9]. Para o seu desenvolvimento foram seguidas seis etapas [10]. $\mathrm{Na} 1^{1}$ a etapa definiu-se o tema a ser pesquisado, que partiu da necessidade de identificar recomendações sobre a participação da enfermagem junto ao paciente com COVID-19, assim, estabeleceu-se a seguinte questão norteadora: Quais as evidências disponíveis na literatura sobre a abordagem aos pacientes com complicações associadas ao novo coronavírus (COVID-19) e quais as principais necessidades humanas básicas afetadas? A busca, a seleção e a avaliação crítica dos estudos foram realizadas em maio de 2020, por dois revisores.

$\mathrm{Na} 2^{\mathrm{a}}$ etapa, houve o estabelecimento de critérios de inclusão e de exclusão. Com este objetivo, definiu-se, como limites das buscas, a inclusão de estudos realizados com seres humanos, publicados na íntegra no último ano, entre 2019 e 2020, devido ao ano de 2019 ter sido o ano da propagação do novo coronavírus na China. Não houve restrição de idiomas. Os estudos foram pesquisados na Biblioteca Virtual Scientific Electronic Library Online (Scielo) Sistema Latino-Americano e do Caribe de Informação em Ciências da Saúde (Lilacs); National Library of Medicine (PubMed) e na Cochrane. 
Para a seleção dos artigos utilizaram-se os descritores 'Respiratory Distress Syndrome', 'Nursing' e 'COVID-19', com o operador boleano 'AND', previamente consultados nos dicionários Medical (MeSH) e Descritores em Ciências da Saúde (DeCS). Na 3ª etapa ocorreu a identificação dos estudos préselecionados e selecionados. Após a combinação dos descritores, emergiram dois estudos do Lilacs, dos quais um foi selecionado e descartado por estar repetido na Pubmed, que somou 139, dos quais oito foram selecionados; a Scielo não apresentou estudos e na Cochrane um estudo, que não atendia os critérios de inclusão (Figura 1). Para a coleta dos dados, utilizou-se um protocolo desenvolvido pelas autoras, com informações sobre aspectos considerados pertinentes como: autores, título do estudo, revista, país, idioma, detalhamento metodológico, amostra, ano de publicação, resultados. Isso permitiu avaliar individualmente os estudos e possibilitou a identificação de similaridades e diferenciações entre eles (Quadro 1).

Os estudos excluídos trouxeram vários aspectos e abordagens sobre 0 impacto da pandemia do novo coronavírus, como: Tratamento farmacológico; Gestante com a COVID-19; Saúde da criança; Dados clínicos dos pacientes; Planejamentos e implicações; Contaminação pelo coronavírus; Manejos específicos; Estudos sobre prevenção; Estudos sobre infectividade; Impacto nos profissionais da área da saúde e Impacto sobre pacientes com doenças crônicas.

$\mathrm{Na} 4^{\mathrm{a}}$ etapa, realizou-se a análise crítica dos estudos selecionados pela aproximação temática das informações apresentadas, a partir da leitura dos títulos e dos resumos, bem como da leitura na íntegra dos estudos. Já na 5a etapa, ocorreu a análise e interpretação dos resultados, com avaliação criteriosa das publicações selecionadas.

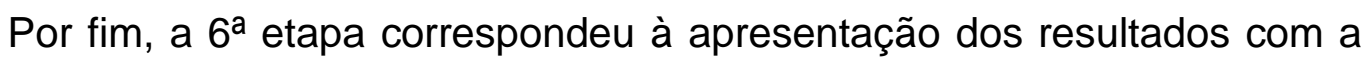
síntese das oito publicações que compuseram a análise e síntese final da revisão de literatura, com isso, os problemas clínicos mencionados nos artigos foram analisados a luz da teoria das necessidades humanas básicas e elencadas as necessidades humanas básicas afetadas, baseadas no referencial conceitual de Wanda Horta. 


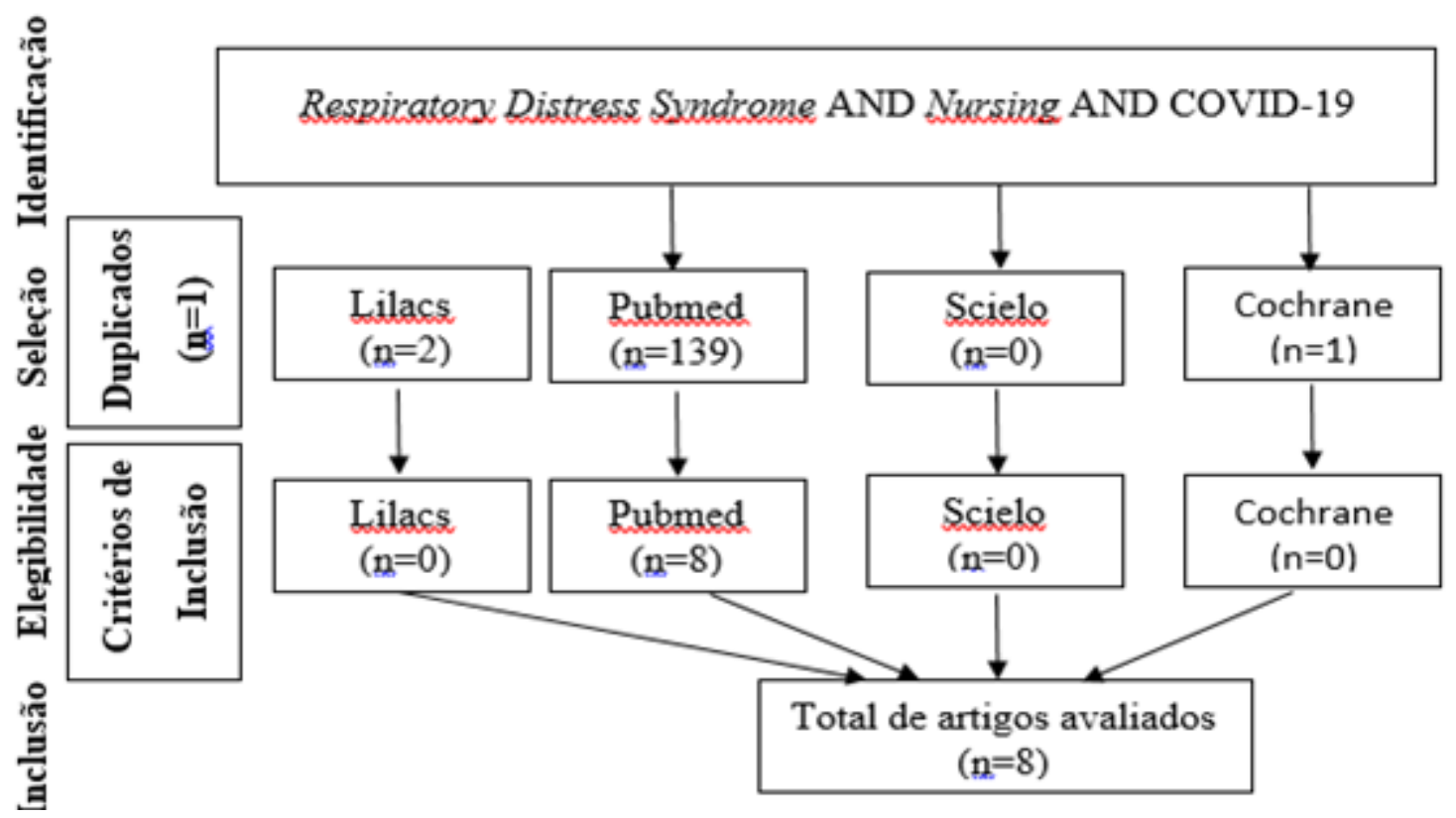

Figura 1 - Artigos incluídos na revisão integrativa, Pelotas/RS, Brasil, 2020.

\section{Resultados}

Caracterização das publicações

Os oito estudos encontrados para esta revisão integrativa foram produzidos por médicos e publicados no ano de 2020 [11-18], cinco (63\%) publicados por intensivistas, anestesistas, nefrologistas e outro por autores da área da terapia intensiva, toxicologia e ortopedia. Majoritariamente produzidos na China, dois na Inglaterra e um no Canadá (Tabela I).

Tabela I - Caracterização dos estudos que compõem a revisão integrativa de literatura, Pelotas/RS, Brasil, 2020 (ver anexo em PDF)

Mesmo com a utilização de descritores bem definidos para a questão norteadora a ser discutida, a atuação da Enfermagem no atendimento não foi evidenciada nos estudos. Portanto, ao relacionar as complicações causadas pelo novo coronavírus foram identificadas as necessidades humanas básicas afetadas, conforme a teoria das necessidades humanas básicas, de Wanda Horta (Tabela II). 
Tabela II - Complicações associadas a COVID-19 e as necessidades humanas básicas afetadas, Pelotas/RS, Brasil, 2020

\begin{tabular}{|c|c|c|}
\hline $\begin{array}{l}\text { Complicações associadas a } \\
\text { COVID-19 }\end{array}$ & Necessidade afetada & NHB \\
\hline Pneumonia [11-14,18] & Oxigenação & Psicobiológicas \\
\hline Dispnéia $[11-14,18]$ & Oxigenação & Psicobiológicas \\
\hline Hipoxemia [12] & $\begin{array}{l}\text { Oxigenação/Orientação no tempo e } \\
\text { espaco }\end{array}$ & $\begin{array}{l}\text { Psicobiológicas/P } \\
\text { sicossociais }\end{array}$ \\
\hline Ventilação mecânica [12,13] & $\begin{array}{l}\text { Oxigenação, Locomoção, Integridade } \\
\text { cutâneo-mucosa, Mecânica } \\
\text { corporal/Segurança }\end{array}$ & $\begin{array}{l}\text { Psicobiológicas } / P \\
\text { sicossociais }\end{array}$ \\
\hline Anorexia [14] & $\begin{array}{l}\text { Nutrição, necessidade de } \\
\text { terapêutica/Autoestima }\end{array}$ & $\begin{array}{l}\text { Psicobiológicas } / P \\
\text { sicossociais }\end{array}$ \\
\hline Hipertermia [14] & Regulação térmica & Psicobiológicas \\
\hline Trombocitopenia [15] & $\begin{array}{l}\text { Regulação vascular, segurança e } \\
\text { proteção }\end{array}$ & Psicobiológicas \\
\hline Linfopenia [16] & Segurança e proteção & Psicobiológicas \\
\hline Insuficiência renal [16] & $\begin{array}{l}\text { Eliminaçåo, nutrição, regulação } \\
\text { eletrolítica/Autoestima }\end{array}$ & $\begin{array}{l}\text { Psicobiológicas } / \mathrm{P} \\
\text { sicossociais }\end{array}$ \\
\hline Desequilíbrio da glicose [17] & Regulação hormonal, nutrição & Psicobiológicas \\
\hline
\end{tabular}

\section{Discussão}

A atuação da enfermagem no cuidado ao paciente com complicações associadas ao novo coronavírus precisa estar amparada por teorias que solidifiquem a assistência prestada. Neste contexto, a teoria das Necessidades Humanas Básica, alinhada ao Processo de Enfermagem que compreende o Histórico de enfermagem, Diagnósticos de enfermagem, Plano assistencial, Plano de cuidados (prescrição de enfermagem), Evolução e Prognóstico podem contribuir para um atendimento sistematizado, que valorize a atuação do enfermeiro, já que cabe a ele avaliar continuamente a resposta do indivíduo à terapêutica aplicada [19].

No que compete as Necessidades Humanas Básicas, das complicações associadas a COVID-19, a psicobiológica é a mais afetada, sobretudo foram identificados potenciais problemas relacionados a oxigenação [11-14,18]. O processo infeccioso pode evoluir para a Síndrome Respiratória Aguda Grave (SARS-CoV-2), dividida didaticamente em: Estágio I - período de incubação assintomática com ou sem vírus detectável; Estágio II - período sintomático não grave com presença de vírus; Estágio III - sintomático respiratório grave com alta carga viral [20]. 
Ademais, nem todos os estudos descreveram sobre pacientes que evoluíram para disfunção respiratória [15-17], assim os pacientes apresentaram sintomas comuns da doença e tiveram repercussões graves como alterações plaquetárias [15], linfopenia durante sessões de hemodiálise [16] e instabilidade eletrolítica [17]. Ao avaliar a necessidade humana afetada a luz da teoria de Wanda Horta, ressalta-se a psicobiológica, no que tange a regulação vascular [15]; regulação imunológica [16]; a eliminação, nutrição, regulação eletrolítica e autoestima [12] e; a regulação hormonal ou nutrição [17]. Isso demonstra a necessidade de avaliação clínica persistente da enfermagem e a importância da implementação da Sistematização da Assistência de Enfermagem (SAE) ao paciente com coronavírus, não apenas para as complicações mais evidentes como as respiratórias.

Para avaliar as evidências de complicações por COVID-19 em indivíduos com desequilíbrio metabólico da glicose, um estudo chinês [17] avaliou dados clínicos de 65 pacientes. A partir desta avaliação pode-se perceber que, tal desequilíbrio, pode estar crucialmente envolvido na infecção pelo coronavírus. $O$ estudo ainda demostra que pacientes que não recebem adequada atenção a patologias pré-existentes estão sujeitos a complicações, evolução negativa do quadro e necessidade de cuidados mais complexos [17]. Nesse sentido, a fim de evitar maiores complicações, a atenção da Enfermagem precisa estar voltada para resgatar esse processo e evitar a deterioração adicional da COVID-19.

Nesta conjuntura, embora os diabéticos não possuam risco aumentado para contrair o coronavírus, eles têm mais risco de complicações caso sejam infectados, principalmente se não possuem um bom controle da glicemia [21,22]. Além disso, aconselha-se que medicamentos como anti-inflamatório e corticoide sejam evitados para o controle dos sintomas da COVID-19, já que podem comprometer o controle da glicose e a função renal [23]. Neste cenário a atuação da enfermagem nas orientações ao paciente e família podem minimizar a automedicação e consequentes prejuízos à saúde. A alteração na taxa de glicose é um problema de enfermagem que pode estar afetado tanto pela regulação hormonal como pela nutrição [17].

$\mathrm{Na}$ China [14], um estudo identificou que a idade média dos pacientes graves foi elevada e com maior probabilidade de apresentar comorbidades crônicas, propensos a desenvolver complicações, como coinfecções por 
bactérias ou fungos. Os sintomas comuns entre esses pacientes graves incluíram hipertermia, anorexia e dispneia, dos $80 \%$ dos casos atendidos na Unidade de Terapia Intensiva (UTI), 52,3\% foram transferidos para enfermarias, visto que os sintomas foram aliviados, e a taxa de mortalidade dos pacientes em UTI foi de $20,5 \%$.

Ademais, um estudo de coorte [15] que acompanhou 383 pacientes admitidos no Hospital Central de Wuhan, na China, com diagnósticos de infecção por COVID-19 apresentou a correlação entre a trombocitopenia e as alterações plaquetárias com a mortalidade dos pacientes. A trombocitopenia na admissão foi associada a uma mortalidade quase três vezes maior que a de pacientes sem trombocitopenia [15], sendo assim, a enfermagem precisa se atentar às doenças pré-existentes que causam plaquetopenia, como púrpura trombocitopênica e hepatopatias, a fim de priorizar o atendimento.

Ainda na China [16], um relato de cinco casos, descreveu a trajetória de pacientes que realizaram diálise com diagnóstico de infecção por SARS-CoV-2. A linfopenia foi identificada em todos os pacientes, fator que pode contribuir diretamente na complicação do quadro clínico destes pacientes, aumentando as chances de óbito [24]. Tal achado enfatiza a necessidade de cuidados durante o manejo destes pacientes, principalmente durante a realização de procedimentos invasivos: prevenção de infecções, utilização de técnicas assépticas para punção e procedimentos necessários e a observação de sinais e sintomas da evolução do quadro de saúde destes.

$\mathrm{Na}$ Inglaterra, um estudo com 52 pacientes com hipoxemia severa destacou a elevada mortalidade de pacientes críticos, 70\% foram tratados com ventilação mecânica, $11 \%$ na posição prona e 17\% precisaram de hemodiálise [12], o estudo destacou a necessidade de profissionais capacitados para adequado manejo nos procedimentos invasivos. Neste cenário, a atuação do enfermeiro neste contexto envolve a aplicabilidade de amplo conhecimento teórico e prático para identificar e manejar os problemas de enfermagem identificados, além do gerenciamento da equipe de enfermagem para melhor atuação frente as complicações.

Para identificar as melhores práticas em relação à intubação e ventilação a um número expressivo de pacientes com COVID-19, autores chineses [13,14] identificaram que a ventilação em decúbito ventral, sedação e analgesia 
adequada são manejos essenciais. Além de seguir rigorosamente as precauções de autoproteção, as estratégias de intubação mais utilizadas em Wuhan incluíram a preparação completa, a pré-oxigenação satisfatória, indução de sequência rápida e a intubação rápida utilizando o videolaringoscópio. Portanto, novamente a oxigenação é afetada e pode ter efeitos psicológicos negativos, como dor, estresse e lembranças de procedimentos invasivos.

De maneira semelhante, um estudo canadense [18] destacou a experiência de tratar um idoso de 68 anos de idade, o qual relatou estratégias de cuidados intensivos para otimizar a oxigenação pulmonar por técnica de pronação com cânula nasal de alto fluxo, abordagem simples para ser realizada em países com poucos recursos, onde técnicas sofisticadas de UTI podem não estar indisponíveis.

O cuidado ao paciente crítico em unidade intensiva necessita da participação ativa da enfermagem, como no caso do estudo que relatou o caso de paciente com opacidades pulmonares bilaterais sugestivas de pneumonia, 0 qual permaneceu com cânula nasal de alto fluxo e foi realizada a técnica de pronação [18]. A construção do conhecimento em enfermagem está interrelacionada entre a teoria, a pesquisa e a prática clínica que afetam de maneira recíproca, logo essa conexão é necessária para a continuidade do desenvolvimento da enfermagem como profissão e como ciência [25].

Neste sentido, a enfermagem precisa valorizar suas ações e ser incentivada a expor sua prática assistencial. Mobilizar o paciente para posição prona demanda atenta participação da enfermagem, e é altamente eficaz no manejo dos pacientes com Síndrome do Desconforto Respiratório Agudo (SDRA), pois ela ajuda na distribuição mais homogênea da impedância pulmonar. Por isso, essa é a posição indicada para os pacientes que necessitam elevar os valores de pressão expiratória final positiva (PEEP) e fração inspirada de oxigênio $\left(\mathrm{FiO}_{2}\right)$, com objetivo de manter uma boa saturação de oxigênio $\left(\mathrm{SaO}_{2}\right)$ [26].

Isso demonstra que a escolha do tratamento depende do quadro geral do paciente, assim como de suas peculiaridades, e disponibilidades do serviço, devendo ser analisado pelas equipes multidisciplinares, compostos por profissionais capacitados para a situação. Nesse sentido, a enfermagem é a equipe que mais está presente durante a acolhida dos pacientes, sendo a 
responsável pelos cuidados diretos e indiretos aos mesmos. Cabe a estes profissionais, a observação e avaliação da evolução do quadro clínico, e no caso das disfunções respiratórias, especificamente, se atentar para a manutenção da saturação de oxigênio, observar sinais de desconforto respiratório, como taquipneia, sinais de confusão mental e apontamento de falências de outros órgãos [27].

Assim sendo, considerando as necessidades psicossociais afetadas destaca-se o enfrentamento da doença, visto que ainda não há um tratamento definitivo para a COVID-19 e como já citado anteriormente, os infectados apresentam diferentes mecanismos de defesa do sistema imunológico $[1,16]$. Logo, a necessidade de segurança poderá gerar ansiedade, medo e estresse nos pacientes e familiares durante o tratamento; tanto em meio hospitalar como no domicílio.

Por fim, observa-se a questão de aprendizagem afetada, também relacionada à educação à saúde. $O$ acesso ao conhecimento permite que a sociedade entenda os motivos e respeite os protocolos adotados atualmente. $O$ enfermeiro como profissional capacitado e atuante em várias frentes de trabalho na pandemia do novo coronavírus tem um papel relevante na sistematização da assistência e no manejo ao paciente com complicações associadas a COVID-19 [28], assim como na educação em saúde àqueles que permanecem em quarentena em suas residências e às famílias neste ambiente tão adverso.

\section{Limitação do estudo}

A invisibilidade da enfermagem em alguns estudos não condiz com a realidade da dedicação diária no processo de cuidar desses profissionais. Portanto, a carência de estudos da enfermagem sobre a participação dela foi um fator limitante para explorar a atuação desta categoria profissional. Contudo, isso não diminui a qualidade dos resultados apresentados, pois eles podem projetar as atividades de enfermagem essenciais ao cuidado das pessoas com coronavírus. 
Conclusão

Ressalta-se a importância de ferramentas como a Teoria das Necessidades Humanas Básicas para a viabilização de um cuidado efetivo ao paciente, que consiga solucionar todas as suas demandas, através da identificação de problemas e efetivação do cuidado, tornando a Sistematização da Assistência de Enfermagem um mecanismo indispensável a profissão.

Assim, por meio desta revisão sistemática integrativa foi possível explorar as complicações associadas ao novo coronavírus e ao manejo ao paciente sintomático e sua evolução sintomatológica. Ademais, reforça-se que apesar da limitação de estudos da enfermagem, esta profissão e sua vigilância no cuidado fazem diferença no percurso da evolução da COVID-19, especialmente, ao paciente crítico.

\section{Referências}

1. Ministério da Saúde. Diretrizes para Diagnóstico e Tratamento da COVID-19. Brasília: Ministério da Saúde; 2020. 398p.

2. Garcia CSNB, Pelosi P, Rocco PRM. Síndrome do desconforto respiratório agudo pulmonar e extrapulmonar: existem diferenças? Rev Bras Ter Intensiva 2008;20:178-

83. https://doi.org.10.1590/S0103-507X2008000200011

3. Simões RCB, Costa GF, Russi ALM, Santamarina AC, Colla LA, Dacia MF. Síndrome do desconforto respiratório agudo: definição de Berlim e Ventilação Protetiva. Revista Científica Multidisciplinar Núcleo do Conhecimento 2018;2(8):40-51.

4. World Health Organization. Novel coronavírus-2019. Technical Guidance. Case Management. Operational considerations for case management of COVID-19 in health facility and community 2020 [citado 2020 Ago 21]. https://www.who.int/publicationsdetail/10665-331492

5. Conselho Federal de Enfermagem. Resolução ${ }^{\circ}$ 358/2009. Dispõe sobre a Sistematização da Assistência de Enfermagem e a implementação do Processo de Enfermagem. Brasília: COFEN; 2009. [citado 2020 Ago 24]. http://www.cofen.gov.br/resoluo-cofen-3582009 4384.html

6. Marques DK. Construção e validação de um instrumento para a implementação do processo de enfermagem em escolares hospitalizados [Tese]. Paraíba: Universidade Federal da Paraíba; 2015. [citado 2020 Ago 28]. 137 p. https://repositorio.ufpb.br/jspui/handle/tede/7638

7. Horta WA. Processo de Enfermagem. São Paulo: EPU; 1979. 
8. Schmitz EL, Gelbcke FL, Bruggmann MS, Luz SCL. Filosofia e marco conceitual: estruturando coletivamente a sistematização da assistência de enfermagem. Rev Gaúcha Enferm 2016;37(spe):e68435. https://doi.org/10.1590/19831447.2016.esp.68435

9. Rodgers BL, Knafl KA. Concept development in nursing: foundations, techniques, and applications. 2nd ed. Philadelphia: Saunders; 2000.

10. Mendes KDS, Silveira RCCP, Galvão CM. Uso de gerenciador de referências bibliográficas na seleção dos estudos primários em revisão integrativa. Texto Contexto Enferm 2019;28:e20170204. https://doi.org/10.1590/1980-265X-TCE-2017-0204

11. Alhazzani W, Møller MH, Arabi YM, Loeb M, Gong MN, Fan E et al. Surviving sepsis campaign: guidelines on the management of critically ill adults with coronavirus disease 2019 (COVID-19). Intensive Care Med 2020;46:854-87. https://doi.org/10.1007/s00134$\underline{020-06022-5}$

12. Yang $\mathrm{X}, \mathrm{Yu} \mathrm{Y}, \mathrm{Xu}$ J, Shu $\mathrm{H}, \mathrm{Xia} J$, Liu $\mathrm{H}$ et al. Clinical course and outcomes of critically ill patients with SARS-CoV-2 in Wuhan, China: a single-centered, retrospective, observational study. Lancet Respir Med 2020;20:S2213-2213. https://doi.org/10.1016/S2213-2600(20)30079-5

13. Meng L, Qiu H, Wan L, Ai Y, Xue Z, Guo Q, et al. Intubation and Ventilation amid the COVID-19 Outbreak: Wuhan's Experience. Anesthesiology 2020;132: 1317-32. https://doi.org/10.1097/ALN.0000000000003296

14. Zhang G, Hu C, Luo L, Fang F, Chen Y, Li J et al. Clinical features and short-term outcomes of 221 patients with COVID-19 in Wuhan, China. J Clin Virol 2020;127:104364. https://doi.org/10.1016/j.jcv.2020.104364

15. Liu Y, Sun W, Guo Y, Chen L, Zhang L, Zhao et al. Association between plate let parameters and mortality in coronavirus disease 2019: Retrospective cohort study. Plaquetas 2020;0(0):1-7. https://doi.org/10.1080/9537104.2020.1754383

16. Wang R, Liao C, He H, Hu C, Wei Z, Hong Z, et al. COVID-19 in hemodialysis patients: a report of 5 cases. Am J Kidney Dis 2020;20:S0272. https://doi.org/10.1053/i.ajkd.2020.03.009

17. Li Z, Liu G, Wang L, Liang Y, Zhou Q, Wu F et al. From the insight of glucose metabolism disorder: Oxygentherapy and blood glucose monitoring are crucial for quarantined COVID-19 patients. Ecotoxicol Environ Saf 2020;197:110614. https://doi.org/10.1016/i.ecoenv.2020.110614

18. Slessarev M, Cheng J, Ondrejicka M, Arntfield R. Critical Care Western Research Group. Patient self-proning with high-flow nasal cannula improves oxygenation in COVID-19 pneumonia. Can J Anaesth 2020;1:1-3. https://doi.org/10.1007/s12630-020$\underline{01661-0}$

19. Vidigal PD, Fuly PSC, Santos MLSC, Camacho ACLF, Souto MD, Santos SC, et al. Main basic human needs affected in patients with cancer and venous thromboembolism: integrative review. Revista Enfermagem Atual 2017; Edição 
Especial. Available from: file://C:/Users/User/Downloads/559-Texto\%20do\%20artigo1751-1-10-20191012\%20(2).pdf

20. Gattinoni L, Copolla S, Cressoni M, Busana M, Rossi S, Chiumello D et al. COVID-19 does not lead to a "typical" acute respiratory distress syndrome. J Respir Crit Care Med 2020;201(10):1299-1300. https://doi.org/10.1164/rccm.202003-0817LE

21. American Diabetes Association. COVID-19 (Coronavirus). 2020 [citado 2020 Jul 23]. https://www.diabetes.org/diabetes/treatment-care/planning-sick-days/coronavirus

22. Sociedade Portuguesa de Diabetologia, Sociedade Portuguesa de Endocrinologia, Diabetes e Metabolismo, Sociedade Portuguesa de Medicina Interna, Associação Portuguesa de Medicina Geral e Familiar. Documento de consenso e abordagem do doente diabético integrado no plano nacional de preparação e de resposta para a doença por coronavírus (COVID-19). 2020 [citado 2020 ago 13]. https://www.spmi.pt/nedm-diabetes-covid-19/

23. Day M. Covid-19: ibuprofen should not be used for managing symptoms, say doctors and scientists. BMJ 2020;368:m1086. https://doi.org/10.1136/bmj.m1086

24. Sociedade Brasileira de Medicina Tropical. COVID-19: doença sistêmica com viés hematológico importante. Brasília: SBMT; 2020. [citado 2020 Jul 26]. https://www.sbmt.org.br/portal/covid-19-systemic-disease-with-significanthematological-bias/

25. Bousso RS, Poles K, Cruz DALM. Conceitos e Teorias na Enfermagem. Rev Esc Enferm USP 2014;48(1):141-5. https://doi.org/10.1590/S0080-623420140000100018

26. Winck JC, Ambrosino N. COVID-19 pandemic and noninvasive respiratory management: Every Goliath needs a David. An evidence based evaluation of problems. Pulmonology 2020;24(4):213-20. https://doi.org/10.1016/j.pulmoe.2020.04.013

27. The Thoracic Society of Australia and New Zealand. National COVID-19 clinical evidence task force. Caring for people with COVID-19. Living Guidelines. 2020 [citado 2020 Jun 24]. https://covid19evidence.net.au/\#living-guidelines

28. Queiroz AGS, Souza RZ, Sottocornola SF, Barbosa SJ, Pinheiro FA, Souza LP et al. Nursing diagnoses according to the NANDA International taxonomy for systematizing nursing assistance to COVID-19. J Health Biol Sci 2020;8(1):1-6. https://doi.org/10.12662/2317-3206jhbs.v8i1.3352.p1-6.2020 